Revue de l'Institut des langues et cultures

d'Europe, Amérique, Afrique, Asie et Australie

41 | 2020

Escrituras nómadas en el mundo hispánico contemporáneo

\title{
Les écritures nomades d'un écrivain nomade, Hernán Casciari
}

Nomadic Writings of a Nomadic Writer, Hernan Casciari

\section{Marina Letourneur}

\section{OpenEdition}

Journals

Édition électronique

URL : http://journals.openedition.org/ilcea/11331

DOI : 10.4000/ilcea. 11331

ISSN : 2101-0609

Éditeur

UGA Éditions/Université Grenoble Alpes

Édition imprimée

ISBN : 978-2-37747-224-6

ISSN : 1639-6073

Référence électronique

Marina Letourneur, «Les écritures nomades d'un écrivain nomade, Hernán Casciari », ILCEA [En ligne], 41 | 2020, mis en ligne le 03 novembre 2020, consulté le 03 février 2021. URL : http:// journals.openedition.org/ilcea/11331; DOI : https://doi.org/10.4000/ilcea.11331

Ce document a été généré automatiquement le 3 février 2021

(c) ILCEA 


\title{
Les écritures nomades d'un écrivain nomade, Hernán Casciari
}

\author{
Nomadic Writings of a Nomadic Writer, Hernan Casciari
}

\author{
Marina Letourneur
}

\section{«Yo es otro»}

1 Nous avons choisi de reprendre là le titre d'un article du blog Orsai publié le 24/02/2005, dans lequel Casciari explique que malgré tous ses efforts pour entretenir les habitudes culturelles et la façon de parler des Argentins - «C'est que le nomade au fond reste attaché à sa terre, produit des mouvements aberrants, si vous préférez il gigote, il se débat, il résiste comme le touareg dans son désert » (Deleuze, $2003: 233$ ) -, au fil des années il s'est peu à peu fondu dans la culture espagnole et a intégré les spécificités idiomatiques de son pays d'accueil.

2 La «déterritorialisation » ${ }^{1}$ de Casciari avait eu lieu environ quatre ans auparavant, lorsque à peine trentenaire, il avait quitté Buenos Aires pour s'installer à Barcelone par amour pour une Catalane. En Argentine, il avait déjà publié deux romans ${ }^{2}$ et collaboré à différents journaux, mais son déracinement allait d'abord l'empêcher d'écrire, avant de provoquer un changement dans sa manière d'écrire. «El viaje como metáfora y síntesis del empezar desde cero ", dirait Aínsa (2012: 86). Par sa dimension globale et parce qu'il offre d'innombrables outils facilitant la communication, Internet a permis à l'écrivain nomade sa "reterritorialisation", autrement dit ce «mouvement qui consiste à refaire le territoire sur quelque chose de différent, d'une tout autre nature» (Deleuze \& Guattari, $1980: 214$ ). Casciari explique comment le blog s'est avéré déterminant dans la poursuite de son activité d'écrivain :

Un día supe que existía un formato sencillo en Internet, desde el que se podía escribir y publicar sin ser un experto en informática. Internet no es España ni es Argentina ni es nada. Se puede escribir voseando o incluso inventar un idioma. Más respeto, que soy tu madre empezó así. A lo bruto y sin mayores secretos: escribir lo que sea para sentirme cerca de un lugar en el que ya no estaba. (Friera, 2015) 
3 Casciari a ainsi rejoint ces écrivains vivant hors de leur pays d'origine, qui imaginent « la patria a la distancia " (Aínsa : 133) et dont « los temas narrativos siguen diagnosticando, a veces con inevitable nostalgia, las sociedades de las que provienen » (Aínsa : 144-145). Avant de devenir Más respeto que soy tu madre, son Weblog de una mujer gorda ${ }^{3}$ a commencé comme une farce destinée à amuser quelques amis restés en Argentine à qui Casciari avait envoyé le lien vers le blog par e-mail. En se mettant dans la peau de Mirta Bertotti, une femme au foyer de 52 ans plus désabusée que désespérée, vivant depuis toujours à Mercedes (petite ville située à une centaine de kilomètres à l'ouest de Buenos Aires), Casciari conjurait le mal du pays en renouant avec ses racines puisqu'il est né et a longtemps vécu à Mercedes et reconnaît s'être inspiré de sa mère pour créer le personnage de Mirta (Casciari, 2012).

4 Le pacte de lecture est le suivant : le lecteur lit les anecdotes presque quotidiennes que Mirta écrit sur son blog avec l'aide de son fils aîné, plus à l'aise qu'elle avec les nouvelles technologies. Dans les premiers articles, qui plantent le décor, la narratrice parle plus des membres de sa famille que d'elle-même (ou de ses kilos superflus) et fait part des diverses difficultés auxquelles elle est confrontée : une situation économique à l'image de celle du pays encore fortement affecté par la crise, un mari au chômage et à la main leste, trois adolescents et un beau-père imprévisibles. Il s'agit d'articles courts, écrits dans un registre de langue courant ou familier qui dénote, à l'instar du titre du blog, un certain franc-parler. Les anecdotes relatées, qui oscillent entre le réalisme, le burlesque et le comique, constituent « una historia costumbrista y delirante » (Wain, 2004). Les Bertotti de Casciari pourraient être les cousins argentins des Simpson de Matt Groening; à travers leurs différentes (més)aventures, l'auteur dépeint le quotidien de la classe moyenne argentine en projetant « una mirada propia y auténtica desde la periferia » (Aínsa : 122).

Le blog a rencontré un succès fulgurant, en Argentine d'abord, puis dans les autres pays hispanophones soit une quinzaine de pays au total, passant des sept lecteurs à qui il était destiné au départ à plusieurs milliers (Wain, 2004). Pourtant, nourri par la nostalgie, il dépeint un univers très argentin :

Los [temas] de Más respeto, que soy tu madre tienen que ver con la memoria emotiva: Mercedes, mi familia, otras familias que conozco, las características de algunos amigos, una forma exagerada de "ser argentino", y las ganas de recordar ciertas expresiones antiguas. (El País, 2005)

6 En effet, Casciari a écrit son blog « con acento y jerga de Argentina » ${ }^{4}$. Aussi, l'évolution du lecteur modèle, au fur et à mesure que le blog gagnait en audience, l'a conduit à ajouter des notes pour clarifier certains mots ou certaines expressions, de manière à la fois didactique et humoristique :

Inicialmente, cada palabra demasiado argentina estaba subrayada con una línea de puntos roja. Cuando un lector foráneo pasaba el cursor por esa palabra, por ejemplo "Alfonsín", aparecía una pequeña definición, también estaba redactada desde el discurso de Mirta: "Este hombre fue un presidente que nos cagó de hambre del '83 al '88 y que después se escapó porque fue siempre un bolastriste". Cosas por el estilo. (Friera, 2005)

7 De la même manière, certains articles mettent en avant des aspects culturels typiquement argentins - comme la recette des beignets à la pâte de coing ou encore le maté - dans le but d'informer les lecteurs étrangers.

D'autres aspects culturels occupent une place de choix, comme le football. Ainsi, Don Américo, fervent supporter de Boca Junior, regrette que son fils Zacarías supporte 
Racing, tandis que celui-ci est soulagé d'apprendre que son fils est homosexuel et non pas supporter de Boca, comme lui avait dit Mirta pour faire diversion. L'article de cette dernière intitulé « Vivir para contarlo ", en date du 21/04/2004, est une lettre adressée à un Maradona entre la vie et la mort, non seulement pour lui rendre un vibrant hommage, mais aussi pour expliquer aux lecteurs pourquoi il est si important pour les Argentins :

Afuera, en la puerta de la clínica donde respirás por un tubito, está lleno de periodistas extranjeros sacándole fotos a un mundo de gente que prende velas y que se pasa la madrugada recitando el rosario. A veces me da un poco de vergüenza que el resto del mundo crea que somos tan básicos, tan cabezones. Pero después me dan ganas de explicarle al mundo que nadie reza por el bocasucia, ni tampoco por el fanfarrón. Me dan ganas de explicarle al mundo qué país es éste, qué pocas alegrías hemos tenido en los últimos veinte años, $y$ que de esas pocas, casi todas vinieron con tu firma. (2012)

9 Tout au long des 200 articles qui composent le blog, il est bien sûr fait allusion à certaines périodes de l'histoire argentine : les vagues migratoires du début du xxe siècle («Gente de buen apellido») et notamment l'immigration italienne - dont est issu Casciari-, incarnée par le personnage du «Nonno» Don Américo qui s'exprime toujours en cocoliche ("Valijas», "Regalos desde Europa»); la junte militaire de 1976 quand Mirta déplore que parmi les célébrités nées à Mercedes figurent deux de ses membres, Videla et Agosti («Meados por los perros »); l'époque de l'hyperinflation, pendant la présidence d'Alfonsín; puis celles de Menem et de De la Rúa. Quant au péronisme, il fait l'objet d'un article entier ironiquement intitulé «Papá, ¿qué corno es el peronismo?». En effet, c'est Mirta, sympathisante du parti radical, qui impose sa définition, ou plutôt son point de vue, tout en moquant l'adhésion de son mari à ce mouvement.

10 La souplesse du format blog a permis à Casciari de se reterritorialiser dans sa ville natale, tout en s'adaptant à ses lecteurs internationaux, particulièrement intéressés par les spécificités culturelles argentines puisque les articles les plus lus du blog, avec le premier et le dernier, ont été ceux où il est fait référence au maté et à Maradona (Clarín, 2008). D'un côté, Casciari semble faire l'inverse de ce que Aínsa constate chez la plupart des écrivains nomades: "En todo caso se trata de evitar de ser latinoamericano como se espera que debe ser para satisfacer el imaginario europeo: lejos de los tópicos y clichés con que siempre se lo ha caracterizado " (Aínsa: 70). Mais d'un autre côté, sa condition d'exilé volontaire lui permet d'aborder certains aspects culturels ou certains clichés sur son pays différemment, en tenant compte des représentations extérieures (sur Maradona, par exemple). Il élabore finalement une "literatura transfronteriza [que] multiplica escenarios y puntos de vista desasida de la noción unívoca de identidad y de patria, incluso proyectando una mirada extranjera sobre el propio solar nativo. " (Aínsa : 68)

11 En 2005, l'auteur reçoit le prix du meilleur blog du monde et des propositions de la part d'éditeurs. Weblog de una mujer gorda devient Más respeto que soy tu madre et «se reterritorialise » dans un format plus classique pour une fiction : le livre, ou plutôt les livres. En effet, selon les pays où il sera édité, l'intermédialité ${ }^{5}$ sera différente, comme nous l'explique l'auteur dans son courrier électronique :

La primera vez que apareció en formato libro fue en España, en donde yo mismo, por sugerencia de mi editora de Plaza \& Janés, adapté o traduje la historia a un contexto español. Al mismo tiempo, la versión original aparecía en Argentina sin cambios, con Editorial Sudamericana. También hubo una edición de Grijalbo en la que, sin consultarme, reprodujeron la versión española con cambios mínimos al universo mexicano. (Por esta y otras razones, renuncié a todas estas editoriales y les quité los derechos de mi obra en 2010.) 
Respecto a las traducciones, todas se basan en la versión original de Argentina (excepto la portuguesa de Editorial Ámbar, que prefirió, por cercanía, el contexto español). La versión francesa de Calmann-Lévy no solo está basada en la original, sino que fue la única en la que participé activamente junto a la traductora, y se consiguió una versión de la que estoy particularmente orgulloso.

12 Bien que la version argentine soit la plus proche du blog d'origine, le passage $d u$ support numérique au papier a forcément entraîné des modifications, ce que souligne Casciari dans le prologue : «[...] es posible que haya pasajes en donde el lector eche en falta un enlace o algún otro elemento muy común en las páginas web e imposible de trasladar al papel » (2012). En effet, le blog était enrichi par des photos (pour accentuer l'effet de réel, Casciari avait posté des photos censées représenter la famille Bertotti), des vidéos, des liens hypertextes, des commentaires de lecteurs, des illustrations de Bernardo Erlich ou encore des surbrillances (nous pensons au lexique typiquement argentin ou aux noms propres, comme "Alfonsín", explicités par Mirta). Tous ces éléments, à l'exception de quelques dessins d'Erlich, ont évidemment disparu dans la version papier, critiquée par certains journalistes (Viola, 2006) parce que forcément moins originale et plus linéaire que le blog. De plus, le pacte de lecture diffère un peu puisque Casciari avait attendu le dernier article du blog pour révéler son identité, tandis qu'il se présente, dans le prologue de la version argentine, comme le compilateur des articles du blog de Mirta, tout en maintenant l'illusion que cette femme a écrit un blog entre 2003 et 2004 avec le soutien technique de son fils. Le résultat s'apparente à un journal puisque les dates et titres des articles sont conservés. Toutefois, les apostrophes aux lecteurs ou certains effets de réel qui rappellent la forme d'origine du récit - on apprend notamment que deux personnages secondaires, Carmencita et Negra Cabeza, lisent le blog de Mirta - le distinguent du journal intime.

13 Concernant l'adaptation à un contexte péninsulaire, comme l'explique plus haut Casciari, elle répond à une demande de la maison d'édition Plaza \& Janés ${ }^{6}$. On peut s'interroger sur la pertinence de ce choix éditorial dans la mesure où le succès du blog démontre que d'autres lecteurs que les Argentins en avaient apprécié la lecture, et le succès de la traduction française corrobore ce point de vue ${ }^{7}$. En cela, ce récit montre bien que « lo americano [...] participa en forma creciente de un universalismo centralizador que ha dejado de ser exclusivamente occidental " (Aínsa: 127). On devine que l'objectif de Plaza \& Janés était d'atteindre un public qui n'avait pas suivi le blog, ce qui visiblement a été une bonne stratégie puisque l'ouvrage a rapidement été édité en poche (collection Debolsillo) et agrémenté de la mention «best seller ». Casciari s'est plié à la volonté de la maison d'édition et a accepté de réécrire, sous une forme romanesque, le blog d'origine. Nous allons voir que cette réécriture révèle à la fois la dé-reterritorialisation ${ }^{8}$ de Casciari en transposant une histoire et des personnages très argentins dans un contexte espagnol, mais aussi les différents compromis inhérents à cette démarche.

14 Aínsa souligne que «La figura del exilio, tanto del forzado como del voluntario, signa [...] el creciente proceso de pérdida de territorio " (114) et l'exilé se retrouve dans une situation d'entre-deux (identités, langues) et de "loyautés multiples » (Ibid.). Casciari publie la version espagnole deux ans après les premiers articles de son blog et cinq ans après son arrivée en Espagne. Dans son article "Yo es otro", il raconte que son esprit "se contamina de pluralidad lingüística» (Casciari, 2005a). Par conséquent, il décide de se charger lui-même de l'adaptation et de la traduction de son blog. Ainsi, les termes et expressions typiquement argentins («trascartón », «cosa de mandinga», " pelotudo», 
"morfar de lo lindo", pour donner quelques exemples) ont été remplacés tandis qu'on en trouve d'autres, typiquement espagnol(e)s : "gilipollas ", " coño ", "para más inri ", «la pasma», "bragas», etc. Les différences linguistiques ne sont plus un frein à la créativité de Casciari comme c'était le cas à son arrivée en Espagne: "Si escribía bombacha no me entendía nadie; si escribía bragas no era yo » (Wain, 2004). Pour l'auteur, c'est aussi une manière de montrer que désormais sa vie de famille est en Espagne, car si les dédicaces de la version argentine étaient pour ses parents, celle de la version espagnole est adressée à sa compagne enceinte de leur fille, qui relisait régulièrement ses chapitres (Casciari, $2006: 7$ ).

L'auteur accepte les consignes de la maison d'édition qui lui demandait un roman et condense le blog en une introduction et 81 chapitres, ce qui implique la disparition de certains épisodes et personnages et une réorganisation du récit. Par souci de cohérence dans la progression de l'intrigue, les chapitres ne suivent pas l'ordre chronologique des articles du blog comme dans la version papier argentine.

16 Le pacte de lecture n'est plus le même et s'avère plus confus. Il n'y a plus de prologue mais une sorte d'introduction dans laquelle la narratrice se présente et présente les membres de sa famille. Elle semble écrire son journal sur un ordinateur puisqu'elle fait, en même temps, des recherches sur internet concernant la ménopause, ce qui est cohérent dans la mesure où l'illustration de Bernardo Erlich, sur la couverture du livre, représente une ménagère en train de taper à l'ordinateur un début de récit qui parodie l'incipit du Quichotte: "En un lugar de mi casa de cuyo nombre no quiero acordarme». Cependant, à plusieurs reprises au long des chapitres suivants, elle dit écrire sur un cahier, lequel ne s'apparente pas à un journal dans la mesure où les chapitres ne contiennent aucune date. Devenu roman, le récit est recentré sur les états d'âmes de la narratrice et les mésaventures des membres de la famille et est forcément moins en phase avec l'actualité que ne l'était le blog.

17 À la demande de la maison d'édition encore, Casciari change le nom de certains personnages : Mirta Bertotti devient Lola B. et son fils Claudio (alias Caio) devient Antonio (Toño). Les autres personnages principaux (Zacarías, Nacho et Sofi) conservent le même nom. Si les personnages principaux gardent les mêmes caractéristiques générales, l'adaptation au contexte péninsulaire conduit toutefois l'auteur à effectuer quelques modifications: Zacarías a perdu son emploi au chantier naval, il supporte le «Depor » (club de football de La Corogne) et affiche des sympathies pour le PP (et pour Franco, lorsqu'il est ivre) alors que Lola vote pour le PSOE. L'adaptation montre parfois quelques limites - de notre point de vue - et s'avère moins pertinente: ainsi Don Américo parle toujours un mélange d'espagnol et d'italien, non plus à cause de ses origines mais en raison de nombreux voyages en Italie par le passé, officiellement pour le travail, officieusement parce qu'il avait une maîtresse là-bas.

L'auteur opère une transposition concernant le personnage de Negra Cabeza en fonction des différentes situations migratoires de l'Argentine et de l'Espagne : l'amante de Caio / Toño puis de Don Américo, immigrée paraguayenne dans la version argentine, devient une immigrée de Guinée Equatoriale dans la version espagnole. Ce choix permet à la fois de refléter l'importance de l'immigration africaine en Espagne au moment de la publication de l'ouvrage tout en justifiant que le personnage parle déjà l'espagnol.

19 Les personnages secondaires sont moins nombreux, dans cette version réduite, cependant l'un d'eux, le cuisinier uruguayen Douglas, prend une place plus importante. 
Sorti de l'intrigue en trois articles dans la version argentine, à cause de l'effet qu'il fait à Mirta et de la jalousie de Zacarías, il est au centre de plusieurs chapitres dans la version espagnole. Casciari fusionne un article où apparaît Douglas avec celui où Mirta évoque sa passion pour le film Sur la route de Madison et maintient un certain suspense en parodiant l'intrigue du film: seule avec Douglas, Lola cédera-t-elle ou non au charme et aux avances d'un homme plus attentionné que le sien? Doit-on y voir une volonté de cibler un public féminin qui peut s'identifier à Lola B. ou à Meryl Streep ? Casciari le laisse entendre, tout en contournant avec humour la question concernant la catégorie d'ouvrage dans laquelle il classerait cette version espagnole de son blog:

A mí me cuesta catalogar el libro dentro de alguna clase de literatura. Es literatura liviana, eso seguro. ¿Pero es de humor? ¿Es sensible? ¿Es para mujeres maduras o para drogadictos inmaduros? Sigo sin saberlo. (El País, 2005)

Évidemment, l'adaptation à un contexte espagnol a conduit l'auteur à supprimer certaines références culturelles propres à l'Argentine ou à effectuer des transpositions, à une exception près : la fête des 15 ans de Sofi, ce qui peut surprendre dans la mesure où cette fête ne se célèbre qu'en Amérique latine ou dans certaines familles latinoaméricaines en Espagne (Gosálvez: 2017). Par ailleurs, certains faits de société sont différemment traités d'une version à l'autre. Ainsi, dans le blog, Mirta évoque une fois la violence de Zacarías à son égard et comment elle y a mis fin très vite (Casciari, 2012 : «La doble llave mortal»). Dans la version espagnole, il n'en est pas question. Peut-être parce que la violence conjugale est alors un sujet sensible en Espagne et qu'il aurait été malvenu de l'intégrer dans un récit humoristique ? Cependant, il n'est pas plus légitime de frapper ses enfants, or Zacarías ne se prive pas de le faire dans les deux versions ("He educado a mis tres hijos a golpes, no me pida que cambie mi sistema pedagógico...", Casciari, 2006 : 175) et dans ce cas de figure, Casciari a réussi, en imaginant des scènes rappelant la slapstick comedy où père et fils ou fille se poursuivent, donnent des coups ou les esquivent, à rendre burlesque ce sujet qui pourrait être très sensible, pris au premier degré. On observe, en revanche, qu'il n'est question de la violence physique des enfants envers leurs parents que dans la version espagnole, lorsque Lola explique que Nacho « es el único que nunca le ha levantado la mano a sus padres » (18).

21 L'objectif du blog d'origine était de divertir les lecteurs à travers les aventures loufoques de personnages caricaturaux et il en est de même pour la version romancée. L'anti-héroïne de cette histoire est très drôle parce que c'est une narratrice atypique (surtout sous le format blog) et bien souvent politiquement incorrecte: elle est légèrement raciste, n'hésite pas à mal parler de ses enfants ou à les frapper, fait un portrait bien peu flatteur de son mari qu'elle est tentée de tromper, etc. On observe néanmoins que Lola B. est une version un peu édulcorée de Mirta Bertotti, qui est plus grossière (Casciari, 2012: «Lávate la boca con jabón») et va plus loin encore dans le politiquement incorrect comme lorsqu'elle encourage sa fille de 14 ans à se filmer à moitié nue sur internet pour sortir la famille de la misère (Casciari, 2012 : «La Sofi quiere una webcam " et "Al final la Sofi nos va a dar de comer»). On peut expliquer ces réajustements entre les deux protagonistes par la volonté de Casciari de montrer qu'il sait s'adapter à l'humour des Espagnols, ce qui n'était pas évident pour lui au début de son installation (Varela Pagliaro, 2014).

Les différentes versions papier du blog d'origine ont été un succès, bien que l'on constate que cette intermédialité a généré une perte plus ou moins importante de l'authenticité de l'œuvre d'origine. Avec ce « journal d'une grosse dame », Casciari a su 
tirer pleinement profit d'internet et du blog pour créer un nouveau genre : le blogroman, auquel nous allons nous intéresser maintenant.

\section{«Orsai »}

Nous avons vu que Casciari a découvert les blogs lorsqu'il était en Espagne où ce format connaissait un grand succès (comme dans le reste du monde " connecté »). Cette forme d'écriture parfaitement adaptée à sa condition d'écrivain nomade lui a permis de se réinventer, de trouver son propre style (Valera Pagliaro, 2014) et la nostalgie pour son pays d'origine a été sa principale source d'inspiration, comme nous venons de le voir avec Más respeto que soy tu madre. Quelques mois après la création de son premier blog, Casciari en a ouvert un autre, personnel, qu'il a choisi de nommer "Orsai", transcription phonétique approximative du terme footballistique " off-side ", parce qu'il se sentait hors-jeu, pas au bon endroit ${ }^{9}$. C'est dans ce blog qu'il a publié, entre autres, les textes rassemblés ensuite dans les ouvrages intitulés, selon les pays où ils ont été édités, España, decí alpiste (Argentine, Sudamericana, 2008) ou España, perdiste (Espagne, Plaza \& Janés, 2007). Ces articles qui traitent de «la conquête » de l'Espagne par les immigrés argentins, nombreux après la crise de 2001, sont fortement empreints d'une nostalgie souvent caricaturale, teintée d'humour et de mauvaise foi.

Aujourd'hui, Casciari n'a plus que son blog Orsai intégré dans son site web (hernancasciari.com), mais il a été l'auteur de plusieurs blogs dont trois fictifs dans le sens où il a longtemps maintenu l'illusion que le narrateur autodiégétique existait réellement comme dans les cas de Mirta (Weblog de una mujer gorda, commencé le 26/09/2003 et achevé le 23/07/2004) ou du voyant Juan Dámaso (Juan Dámaso -vidente ,- 2005), ou a usurpé l'identité d'une personne réelle comme Letizia Ortiz, au moment de l'annonce de son mariage avec l'actuel roi d'Espagne (Diario de Letizia Ortiz, 2004). Le second et le troisième n'ont pas connu le même impact médiatique que le premier, sans doute parce que Casciari ne les alimentait pas avec la même régularité, ce qui n'empêche pas de les intégrer dans la catégorie blog-roman. En réalité, le blog fictif de Letizia Ortiz était une sorte de cheval de Troie créé par Casciari pour attirer un nouveau profil de lecteurs (ou plutôt lectrices) puisqu'il y faisait surtout la promotion de son blog Mujer gorda (El Mundo, 2005). Le succès de ce dernier, en revanche, a valu à son auteur d'être considéré comme le précurseur d'un nouveau genre. Ainsi, pour son numéro du dernier trimestre 2005, la revue électronique TELOS lui a demandé un article qu'il a intitulé "Un acercamiento estructural a la blogonovela». Casciari y propose une définition :

[...] la blogonovela (como género literario) es una historia de largo aliento escrita en capitulos inversos, atomizados, narrados en primera persona, con una trama que ocurre en tiempo real, en donde el protagonista es consciente del formato que utiliza y en el que la realidad afecta al devenir de los acontecimientos. En términos estéticos, la blogonovela es un arte conjunto en el que predominan tres elementos que poseen idéntico valor: la escritura tradicional, el diseño multimedia y la programación informática. (2005b)

Cette définition correspond complètement à son Weblog de una mujer gorda et ses quelques deux-cents articles qui comprenaient des faits d'actualité (par exemple le black-out du 28/09/2003 en Italie évoqué dans l'article "Cortes de luz» en date du 01/10/2003, ou l'hospitalisation de Maradona). Contrairement à la version papier, les chapitres apparaissaient du plus récent au plus ancien et étaient aussi accessibles de façon plus indépendante en cliquant sur leur titre. En outre, Casciari a su tirer profit au 
maximum de ce support informatique en intégrant toutes sortes d'éléments additionnels (photos, dessins, liens hypertextes).

Dans le même article, Casciari énumère un peu plus loin les six caractéristiques essentielles ${ }^{10}$, selon lui, de la structure d'un blog-roman :

1. La blogonovela está escrita en primera persona. [...]

2. La trama ocurre siempre en tiempo real. [...]

3. El protagonista se reconoce como gestor del formato. [...]

4. La realidad afecta al devenir de la trama. [...]

5. El protagonista existe fuera de la trama. [...]

6. El autor no aparece mencionado. [...] (2005b)

Si l'on compare avec le roman en général, on observe d'abord les contraintes qu'impose le format blog quant au choix du narrateur et à son rôle (points 1 et 3) ou encore au temps (points 2 et 4 ) puisque le présent règne en maître (hic et nunc): le récit est forcément publié le jour où il est écrit et ne peut rester étranger à certains faits d'actualité. Ces deux composantes narratives sont indispensables pour que se produise " une suspension consentie de l'incrédulité » (Coleridge, 2013 : 379) chez le lecteur et qu'il accepte le fait qu'il est en train de lire le blog d'une personne qui pourrait exister. Ensuite, les points 5 et 6 permettent de distinguer complètement le blog-roman du roman. Certes, un livre peut être publié anonymement ou sous un pseudonyme, mais dans le cas d'un blog-roman l'auteur doit s'effacer au point de donner l'illusion que le narrateur et l'auteur ne sont qu'une seule et même personne. Par conséquent, le blog est propice à la métafiction dans la mesure où il devient possible pour les lecteurs de communiquer directement, via les commentaires, avec le pseudo auteur du blog, ce qui fut le cas pour Mujer gorda :

Cada capitulo cuenta con un sistema de comentarios, donde ellos [los lectores] hablan literalmente con Mirta, la protagonista. La aconsejan, la retan, la compadecen. Una de las mayores sorpresas para mí, al escribir esta novela, fue la creación espontánea, una regla tácita, jamás escrita ni por mí ni por mis lectores, en donde nadie habla nunca con el autor. Nadie me da pelota: todos conversan con Mirta. (Friera, 2005)

Ce commentaire de Casciari montre aussi que les six caractéristiques du blog-roman qu'il énumère sont empiriques dans le sens où elles découlent de sa première expérience en tant qu'auteur de blog-roman.

Les lecteurs ne se contentent pas de communiquer avec Mirta, mais échangent aussi entre eux et leur participation au processus créatif est très importante pour Casciari :

Para escribir historias en Internet hay que entender que uno es como un anfitrión, que recibe gente en su casa. El anfitrionazgo es vital para que los lectores se sientan cómodos, y también para que sepan que existe una coordinación tácita en el juego de la lectura compartida. No se trata de escribir y publicar. Lo más atractivo está en lo que se genera alrededor de cada texto, que puede ser una continuación o una modificación colectiva que no habías imaginado. (Wain, 2004)

L'auteur s'efface même, parfois, pour laisser la place aux lecteurs, devenus des collaborateurs actifs :

[...] cuando lectores de todo Hispanoamérica comenzaron a conversar entre ellos en los comentarios, dejé de redactar explicaciones, porque me resultó mucho más divertido que un lector colombiano le preguntara a un lector argentino "¿qué quiere decir concheta?", y el argentino le contestase "una chica de clase alta", y un español a su vez explicara "aquí les decimos pija", y que el resto de los latinoamericanos se riera y acabaran todos haciendo chistes sobre cómo les gusta la pija a las conchetas. (Friera, 2005) 
31 Attentif aux réactions de ses lecteurs (« utilizo las charlas entre los lectores para saber por dónde debo ir. ", Ibid.), l'auteur réagit, par exemple, aux commentaires de ceux qui sont choqués par la façon de s'exprimer de Mirta dans l'article du 16/01/2004 intitulé "Lávate la boca con jabón », où la narratrice est outrée par le langage de sa fille tout en étant elle-même très vulgaire dans sa façon de s'exprimer.

Cette interaction avec les lecteurs est bénéfique pour l'auteur, même s'il s'agit parfois de critiques, car elle lui permet de s'améliorer. Dans l'article «Los bloggers muertos no van al cielo " où il compare les ateliers d'écriture et les blogs, il considère que ces derniers sont beaucoup plus formateurs et déclare :

Si hubiera que creer en algo alrededor de la creación literaria y sus secretos, yo solamente creería en dos verdades: desde siempre, en el decálogo del buen cuentista que escribió Horacio Quiroga a principios del siglo pasado; $y$ desde este siglo, en publicar textos online, más o menos todos los días, para que los lean y comenten un grupo de desconocidos de cualquier parte del mundo. No hay nada mejor para mejorar tu prosa que alimentar un blog. (Casciari, 2010 : 157)

La vogue des blogs au début des années 2000 a engendré une sorte d'endogamie dans le sens où des blogs ont été créés pour parler d'autres blogs. C'est en en parcourant quelques-uns que Casciari a pu lire, parfois, des critiques acerbes sur Mujer gorda, comme il le raconte dans son article «Los cinco críticos feroces » (45-50). L'auteur a cependant su tirer profit de l'aspect «work in progress » du blog pour répondre à plusieurs de ses détracteurs - et notamment à un critique de blogs prénommé Borjamari ${ }^{11}$, adepte des titres "à rallonge »- en créant un personnage de croque-mort homonyme, amant éphémère de Nacho, qui s'exprime comme les Espagnols. Dans l'article « Una cena demasiado larga » (publié le 27/11/2003), l'auteur parodie certains de leurs commentaires en imaginant une scène de dîner en famille qui dégénère après que Mirta a demandé à Borjamari ce qu'il avait pensé de sa pizza (qui, bien sûr, fait office de métaphore du blog) :

-Pizza de champiñones Bertotti, o cómo destruir sabores nobles mezclándolos de forma arbitraria para que parezca un entramado gastronómico del discutible gusto popular.

- ¿Eh? - todos nos miramos sin entender.

-Esperad que no he acabado -dice el Borja-; eso era el título. Ésta no es una pizza al uso, suponiendo que exista una definición general para un concepto tan abstracto como la pizza, pero lo que sí está claro es que es toda una apuesta hacia el más pésimo gusto. Si aceptamos la infalibilidad del representante de Dios en la tierra, por supuesto en cuestiones culinarias nada más, esta pizza es infumable. [...]

- ¿Entonces no te gustó la pizza, nene? - digo yo un poco desencantada.

-Teniendo en cuenta que todos vosotros fingís tener una pizzería en Argentina, cuando en realidad sois una agencia de publicidad que está intentando imponer una novela en el mercado editorial español, debo reconocer que por lo menos habéis preparado la comida vosotros mismos. [...]

-Venga ya, mujer -se incorpora el Borja mirándome muy raro-, diga la verdad: usted no es Mirta Bertotti, es un conjunto de autores catalanes, y estas paredes son falsas, todo es un decorado, ¡todo es falso! ¿Por qué quitó las estadísticas la semana que vendió menos de mil pizzas al día? Todos vosotros estáis obsesionados conmigo, ¡todo esto es falso, es una agencia de publicidad catalana! (Casciari, 2012)

Le personnage finit neutralisé (ou plutôt ridiculisé) après une séance d'hypnose puis disparaît du récit. Dans la version romancée destinée au marché espagnol, les traces du blog ont toutes disparues, à l'exception de celle-ci. Il s'agit sans doute d'un pied-de-nez de l'auteur à ses détracteurs puisqu'il a bel et bien fini par imposer son roman sur le marché éditorial espagnol... 

d'être «blogger » et que cette catégorisation liée au support d'écriture qu'il a choisi le dérange :

El siglo Xx era maravilloso: no importaba dónde escribieras, ni en qué soporte; siempre serías un escritor.

Pero a finales del año 2003, intentando mantener mi equilibrio cotidiano con el progreso, empecé a escribir una novela online, y en lugar de utilizar un cuaderno, o una pizarra, o un bolígrafo, o una Olivetti, utilicé un blog.

Desde ese día suena el teléfono en casa y la gente pide hablar con un bloguero. Desde entonces sale mi nombre en la prensa precedido de la palabra blogger. (2010:18)

Certes, le support est nouveau et offre d'autres possibilités que le roman traditionnel, comme nous venons de le voir. Mais le genre n'est pas nouveau car les aventures de Mirta s'inscrivent dans la tradition des romans-feuilletons apparus dans la presse au xIX ${ }^{e}$ siècle. Il n'y a pas si longtemps encore, certains ont même connu un grand succès au point de devenir des livres mondialement connus, comme Les chroniques de San Francisco de Maupin (publiées d'abord dans le San Francisco Chronicle, dans les années 1980) ou le Journal de Bridget Jones de Fielding (1996) publié initialement dans The Independant. Casciari reconnaît au blog l'avantage de permettre une interaction avec les lecteurs, mais elle existait déjà avant les blogs, certes de façon plus limitée dans le temps puisqu'il était alors plus difficile de revenir sur un article de journal longtemps après sa publication que sur un article de blog, disponible en un clic. On pense notamment à un autre écrivain et journaliste argentin, Arlt, qui encourageait lui aussi le dialogue avec les lecteurs de ses Eaux-fortes de Buenos Aires. Il n'hésitait pas à inclure et à commenter des extraits de lettres qu'il recevait, comme dans l'eau-forte: "Me escriben 'simpatizantas' " $(1998: 312)$ ou à rendre hommage à ces lecteurs qui deviennent des collaborateurs quand l'écrivain est en panne d'inspiration, comme dans l'eau-forte publiée le 07/12/1928 et intitulée «El lector que manda tema para crónicas » : « Cada carta con una propuesta sobre qué escribir es como una ayuda gratis y oportuna en esta tarea de yugar a diario. » (Saítta, 1993: 65) Notons que cette courte citation reflète parfaitement le style arltien, marqué par un registre souvent familier et l'emploi du lunfardo ( «yugar »), et cette volonté de l'auteur - que l'on retrouve chez Casciari - de parler le même langage populaire que ses lecteurs.

Le phénomène "blog » s'est considérablement essoufflé aujourd'hui car beaucoup ont disparu peu après l'apparition de réseaux sociaux comme Facebook ou Twitter, ou de plateformes comme Youtube. Casciari a tout de même conservé Orsai, mais semble avoir quelque peu abandonné ce support: les articles sont archivés jusqu'en 2015 et le dernier article publié date du 27/11/2018. Il a néanmoins longtemps écrit à partir de ce blog, c'est-à-dire en direct et en interaction totale avec les lecteurs qui pouvaient accéder à ses brouillons, comme il le précise sur son site pour certains de ses ouvrages, notamment son roman El pibe que arruinaba las fotos (2009) ou ses compilations d'articles : Messi es un perro y otros cuentos (2015) ou El mejor infarto de mi vida (2017). Orsai n'a pas disparu mais a connu une évolution et est devenue une entité culturelle à part. En effet, à partir de ce blog, Casciari a créé à la fois une revue culturelle sans aucune publicité (le premier numéro est sorti en janvier 2011) et une maison d'édition indépendante homonymes. Autrement dit, son premier blog est devenu un phénomène d'édition, mais à partir d'un autre blog, Casciari a pu créer, en plus d'autres ouvrages, une revue papier vendue en pré-commande depuis son site, puis disponible, après sa publication, gratuitement sous format $\mathrm{pdf}^{12}$. En outre, tout ce qu'il a publié depuis Más 
respeto que soy tu madre (version argentine) est disponible sous format papier et édité désormais par Orsai. De l'esprit du blog, l'auteur a conservé la possibilité de s'autopublier et le contact étroit avec les lecteurs, très précieux à ses yeux :

[...] mis lectores son todo el capital de mi oficio. No solamente me importa lo que digan, sino que intento conversar con ellos, ir a visitarlos, apostar boludeces en donde siempre pierdo yo; jugar como se juega en los recreos. De alguna manera, sé que ellos me formaron como narrador y me siento cómodo en esa compañía. (Playo, 2015)

Casciari est un auteur populaire ${ }^{13}$, qui s'exprime souvent de manière populaire. Il cultive autant que possible une proximité virtuelle ou réelle avec ses lecteurs. De nouveau installé en Argentine depuis 2015, il se consacre essentiellement à l'écriture et à la lecture de ses récits à la télévision (Telefé) ou à la radio ${ }^{14}$ mais aussi dans des salles ou théatres du pays ${ }^{15}$ et même hors des frontières ${ }^{16}$, parfois en duo avec un dessinateur ou un musicien, par exemple. Il profite de la proximité physique avec le public pour observer en direct ses réactions :

Lo que busco [...] es que la gente se esté cagando de risa, se relaje, y yo pueda pegarle un palazo en la cabeza justo en ese momento. Que no se den cuenta de nada, que empiecen a moquear sin saber por qué. No siempre sale, pero cuando sale me da la impresión de que es un momento hermoso. (Playo, 2015)

Ainsi, l'écrivain déterritorrialisé, déraciné et sédentaire derrière un écran d'ordinateur, qui se reterritorialisait dans son pays d'origine via un blog, est devenu une sorte d'aède, un conteur nomade comme au temps jadis.

\section{BIBLIOGRAPHIE}

AINSA Fernando (2012), Palabras nómadas. Nueva cartografía de la pertenencia, Madrid : Iberoamericana Vervuert.

ARLT Robert (1998), Aguafuertes, Buenos Aires : Losada.

CASCIARI Hernán (2004), «El viejo folletín y las nuevas tecnologías », en ligne sur Hernán Casciari : $<$ https://hernancasciari.com/blog/articulo/el_viejo_folletin_y_las_nuevas_tecnologias> (18/08/2019).

CASCIARI Hernán (2005), « Yo es otro », en ligne sur Hernán Casciari : <https://hernancasciari.com/ blog/articulo/yo_es_otro> (18/08/2019).

CASCIARI Hernán (2005), « Un acercamiento estructural a la blogonovela », en ligne sur Telos : $<$ https://telos.fundaciontelefonica.com/archivo/numero065/un-acercamiento-estructural-a-lablogonovela/> (18/08/2019).

CASCIARI Hernán (2006), Más respeto, que soy tu madre, Barcelone : Plaza \& Janés.

CASCIARI Hernán (2008), « Charla con los lectores de Orsai », en ligne sur Hernán Casciari : <https:// hernancasciari.com/blog/articulo/charla_con_los_lectores_de_orsai> (18/08/2019).

CASCIARI Hernán (2010), El nuevo paraíso de los tontos, Barcelone : Plaza \& Janés.

CASCIARI Hernán (2012), Más respeto que soy tu madre, Buenos Aires : Orsai (version Kindle). 
CLARIN (2008), « Cómo se construyó Más respeto que soy tu madre », en ligne sur Clarín : <https:// www.clarin.com/ultimo-momento/construyo-respeto-madre_0_Hy5wnRptx.html> (18/08/2019). COLERIDGE Samuel Taylor (2007), « Autobiographie littéraire », La Ballade du vieux marin et autres textes (J. Darras, trad.), Paris : Gallimard.

DELEUZE Gilles \& GUATTARI Félix (1980), Mille plateaux, Paris : Éditions de Minuit.

DELEUZE Gilles (2003), Pourparlers, Paris : Éditions de Minuit.

EL MOSTRADOR (2005), Hernán Casciari: Mujer Gorda es una historia terriblemente latinoamericana, en ligne sur <https://www.elmostrador.cl/noticias/mundo/2005/11/26/hernan-casciari-mujergorda-es-una-historia-terriblemente-latinoamericana/> (18/08/2019).

EL MUNDO (2005), Encuentros digitales: Hernán Casciari, en ligne sur <https://www.elmundo.es/ encuentros/invitados/2005/10/1709/> (18/08/2019).

EL PAIS (2005), Entrevista con Hernán Casciari, en ligne sur <https://elpais.com/cultura/2009/09/25/ actualidad/1253876400_1253882084.html> (18/08/2019).

ESCUELA DE PERIODISMO UAM (2012), "Al comienzo, la cultura no tiene nada que ver con el dinero », en ligne sur El País : <https://elpais.com/cultura/2012/01/10/actualidad/

1326150004_850215.html> (18/08/2019).

FRIERA Silvina (2005), « No sé cómo llegué al segundo capítulo », en ligne sur Página/12:<https:// www.pagina12.com.ar/autores/5997-silvina-friera> (18/08/2019).

GOSÁlvEZ Patricia (2017), « Las fiestas latinas de 15 años arrasan en España », en ligne sur El País : <https://elpais.com/politica/2017/02/07/actualidad/1486485272_700545.html> (18/08/2019).

MÉCHOULAN Eric (2017), « Intermédialité ou comment penser les transitions », en ligne sur Fabula : <http://www.fabula.org/colloques/document4278.php> (18/08/2019).

MIGOZZI Jacques (1997), Le roman populaire en question(s), Limoges : PULIM.

PLAYO José (2015), « Hernán Casciari: el último blogger con todas las letras », en ligne sur La Voz : $<$ https://www.lavoz.com.ar/ciudad-equis/hernan-casciari-el-ultimo-blogger-con-todas-lasletras> $(18 / 08 / 2019)$.

SAITTA Silvia (1993), « Roberto Arlt y las nuevas formas periodísticas », Cuadernos hispanoamericanos (11), Madrid.

SILVA PARANHOS Ana Lucía (2014), « Dé-re-territorialisation », Z. Bernd et N. Dei Cas-Giraldi (dir.), Glossaire des mobilités culturelles, Bruxelles : P.I.E. Peter Lang, 109-126.

VARELA PAGLIARO Nando (2014), « Estoy más contento con que me lea él que nunca leyó y no el crítico de La Nación », en ligne sur Medium : <https://medium.com/@varelapagliaro/estoy-mascontento-con-que-me-lea-el-que-nunca-leyo-y-no-el-critico-literario-de-la-nacion-aa0e2f6c7b65> $(18 / 08 / 2019)$.

Viola Liliana (2006), « Cuando la forma altera el producto », en ligne sur Página/12 : <https:// www.pagina12.com.ar/autores/875-liliana-viola> (18/08/2019).

WAIN Martín (2004), « Lectura en capítulos », La Nación, Buenos Aires. 


\section{NOTES}

1. Entendue comme «mouvement par lequel "on" quitte le territoire » (Deleuze \& Guattari, 1980 :

634). Le nomade est « le Déterritorialisé par excellence » (473).

2. Subir de espaldas la vida (Prix du roman lors de la Biennale de Buenos Aires en 1995) et Nosotros lavamos nuestra ropa sucia (Prix Juan Rulfo en 1998).

3. Le blog, renommé ensuite Mujer gorda, n'est plus accessible en ligne. Nous ferons donc référence soit à l'édition argentine d'Orsai (version Kindle) soit à l'édition espagnole de Plaza \& Janés. Pour les références à la version Kindle, nous indiquerons la date et / ou les titres des chapitres.

4. Correspondance électronique avec l'auteur (04/04/2019).

5. Selon Méchoulan (2017): "l'intermé dialité peut désigner les déplacements, échanges, transferts ou recyclages d'un média bien circonscrit dans un autre ». Précisons que le blog a également été adapté au théâtre par Antonio Gasalla et joué en 2009.

6. Plaza \& Janés et Sudamericana sont deux filiales du groupe éditorial Random House Mondadori.

7. Sans oublier l'intérêt d'autres pays comme l'Allemagne ou la Russie pour une traduction du blog (El Mostrador, 2005).

8. «La vie est ainsi envisagée en un constant mouvement de dé-re-territorialisation: en déplacement répété d'un territoire à l'autre, nous en abandonnons et en fondons de nouveaux » (Silvia Paranhos, 2014 : 114).

9. «A veces, cuando no entiendo un chiste, cuando un código argentino no se desata en mi cabeza con la soltura de la cotidianidad, me siento terriblemente aislado, contrariado, perdido y caducado como una natilla en la nevera. Vencido como un sandy en la heladera. En orsai. En off-side. » (Casciari, 2005a)

10. Nous reprenons seulement les intitulés.

11. Voir : les articles publiés en octobre et novembre 2003 sur le blog de Borjamari, en ligne sur : <https://borjamari.blogspot.com/2003_10_01_borjamari_archive.html > et < https:// borjamari.blogspot.com/2003_11_01_borjamari_archive.html>

12. Le fonctionnement de la revue Orsai est expliqué dans une interview avec l'école de journalisme de l'Université Autonome de Madrid publiée dans El País (2012). Très récemment, Casciari en a parlé au début du programme de radio Perros de la calle du 16/08/2019, disponible à travers ce lien : <https://www.youtube.com/watch?v=Y964D5OpGZI> (19/08/2019).

13. La production littéraire de Casciari correspond à cette définition du roman populaire que propose Migozzi :

«Fiction écrite de masse, qu'on la nomme roman populaire, paralittérature, littérature de grande consommation, littérature sérielle... qu'importe le flacon, pourvu qu'on ait l'ivresse !» (Migozzi, $1997: 12)$

14. Les vidéos et podcasts sont disponibles depuis son site web.

15. Ces rencontres sont parfois gratuites (comme celle qui eut lieu à la cité universitaire de Córdoba le 08/10/2015). Lorsqu'elles sont payantes, le public réserve via l'onglet «Eventos » de son site.

16. Il a fait une tournée en Europe (Espagne, France, Royaume-Uni, Allemagne, Italie) en novembre 2019 et se produira aux États-Unis et au Mexique en mai 2020. 


\section{RÉSUMÉS}

L'Argentin Hernán Casciari s'est fait connaître au début des années 2000 grâce à son blog Mujer gorda, lauréat en 2005 du prix du meilleur blog du monde décerné par la chaîne allemande Deutsche Welle.

La rédaction du blog fictif d'une ménagère argentine, Mirta Bertotti, a débuté peu de temps après l'installation de Casciari à Barcelone, dans le but de divertir ses amis argentins. Son succès ayant considérablement élargi son public de lecteurs, il a été traduit et publié sous format papier dans de nombreux pays, mais aussi réadapté, à la demande de la maison d'édition espagnole Plaza \& Janés, sous la forme d'un roman intitulé Más respeto que soy tu madre, dont la narratrice est devenue Lola B., une femme au foyer espagnole. À partir de cette œuvre qui illustre ce que Aínsa appelle la «transterritorialidad de la narrativa actual » (2012: 69), nous proposons d'analyser, dans un premier temps, les processus de déterritorialisation et de reterritorialisation qui ont opéré au cours de la création du blog puis de sa réécriture destinée à un public espagnol.

Dans un deuxième temps, nous nous intéresserons au blog, que Casciari définit comme "una fusión entre el viejo folletín y las nuevas tecnologías" (2004). Précurseur dans l'utilisation du blog comme espace de fictions, Casciari n'a pas manqué de souligner les avantages, à ses yeux, de cet outil moderne et a même proposé quelques bases théoriques sur la structure d'un blog-roman. En nous appuyant sur les réflexions de l'auteur mais aussi sur son parcours d'écrivain nomade, nous verrons que plus qu'une écriture nomade, le blog semble avoir été une écriture transitoire, à la merci des avancées technologiques.

Argentine writer Hernán Casciari became famous in the early 2000s thanks to his blog Mujer gorda which was awarded the world best blog by German channel Deutsche Welle in 2005.

The fictional blog of Mirta Bertotti, an Argentinean housewife, was written shortly after Casciari moved in Barcelona, in order to entertain his Argentine friends. The success of the blog considerably expanded the number of its readers, so it has been translated and has become a printed book in many countries, and then, at Spanish Plaza \& Janés publisher's request, it has been re-adapted as a novel entitled Más respeto que soy madre, whose narrator became Lola B., a Spanish housewife. This fiction is an illustration of the "transterritorialidad de la narrativa actual" (Aínsa, 2012: 69). First, we suggest to examine the processes of deterritorialization and reterritorialization that operated when the blog was created, and then how it was rewritten for the Spanish readers.

Secondly, we will analyse the blog itself that Casciari defines as "una fusión entre el viejo folletín y las nuevas tecnologías". As a forerunner of the use of the blog as fictional space, Casciari considerably highlighted the benefits to using that modern tool, and he even suggested some theoretical bases on the structure of a blog-novel.

Based on the author's contemplations but also on his career as a nomadic writer, we will see that, more than a nomadic writing, the blog seems to have been a transition writing, highly exposed to technological advances.

\section{INDEX}

Mots-clés : Hernán Casciari, nomadisme, blog-roman, intermédialité

Keywords : Hernán Casciari, nomadism, blog-novel, intermediality 
AUTEUR

MARINA LETOURNEUR

Le Mans Université, 3L.AM 